\title{
Geschichte und technische Möglichkeiten der winkelstabilen Osteosynthese - eine Bildergeschichte
}

\author{
History and Technical Possibilities of Angle-Stable Osteosynthesis - A Pictorial Story
}

\section{Dankward Höntzsch, Stephan Perren, Röbi Frigg}

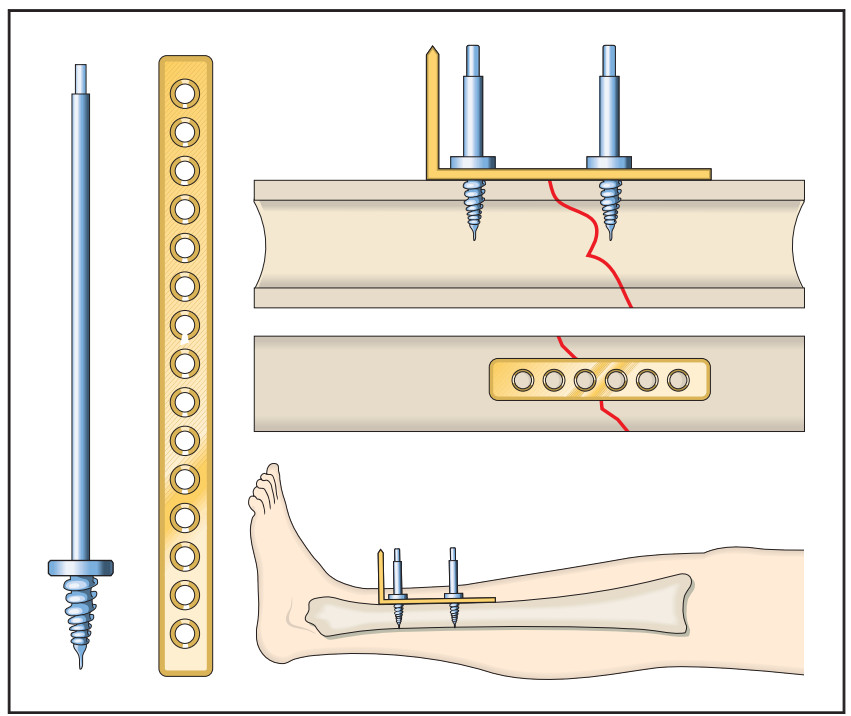

Abb. 1 Auszug aus der Veröffentlichung von Hansmann (1886). Eine Fraktur wird mit einer kurzen Platte gehalten. Die Schrauben sind darin stabil verankert.

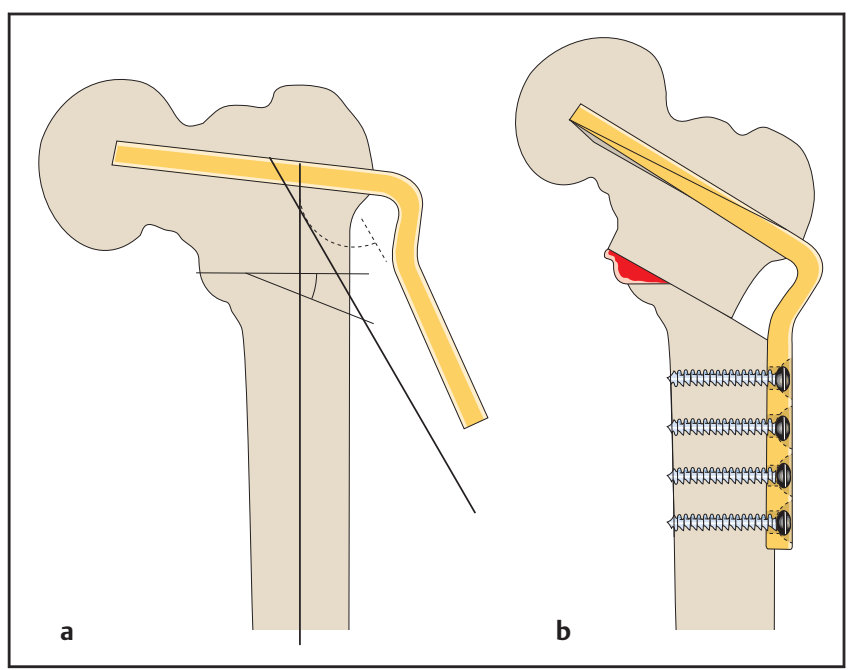

Abb. 3 Klingenplatte (1959). Die (hoffentlich) allen bekannte Kondylenplatte ist ein Beispiel für die große Familie der Winkelplatten $\left(95^{\circ}\right.$, $110^{\circ}, 130^{\circ}$, gerade, gekröpft).

OP-JOURNAL 2012; 28: 124-128

(c) Georg Thieme Verlag KG Stuttgart · New York DOI http://dx.doi.org/10.1055/s-0032-1315299

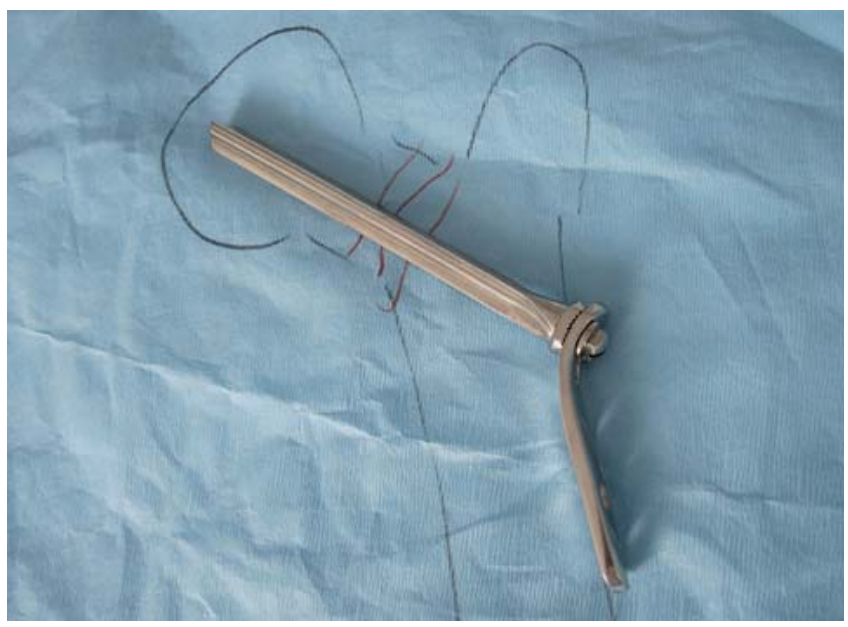

Abb. 2 Pohl'sche Laschenschraube für den proximalen Femur (1940). Die sternförmige Klinge hält den spongiösen Femurkopf. Diese sternförmige Klinge ist mit der Lasche, d.h. Platte, in einem bestimmten Winkel winkelstabil verbunden.

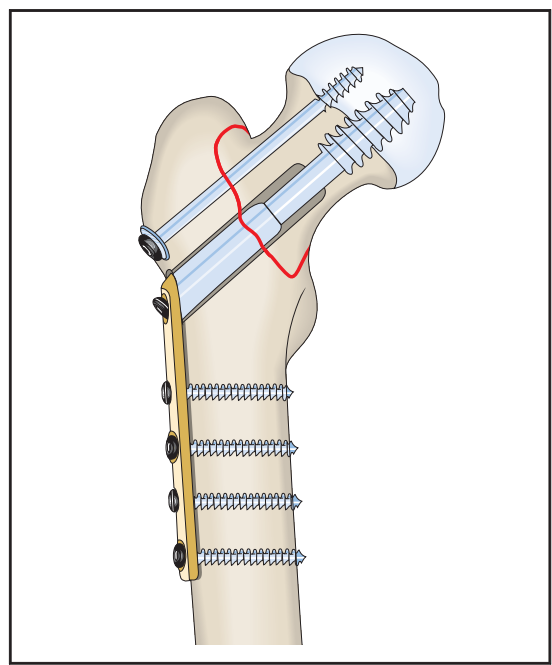

Abb. 4 DHS (1980). Die DHS verwirklicht das Prinzip winkelstabile Stabilisierung wie eine $125^{\circ}$ - bis $135^{\circ}$-Kondylenplatte (siehe Abb. 3). Der Hüftkopf selbst wird mit einer großvolumigen Schraube gefasst. Als weitere Komponente ist hinzugefügt, dass diese Schraube im immer gleichen Winkel, d.h. winkelstabil, nach peripher rutschen kann. So wird einer Last, nämlich dem Körpergewicht, dynamisch nachgegeben, bis die Frakturzonen so aufeinander stehen, dass sie bei der Frakturstabilisierung helfen. In diesem Fall gewinnt man durch Nachgeben Kraft. 


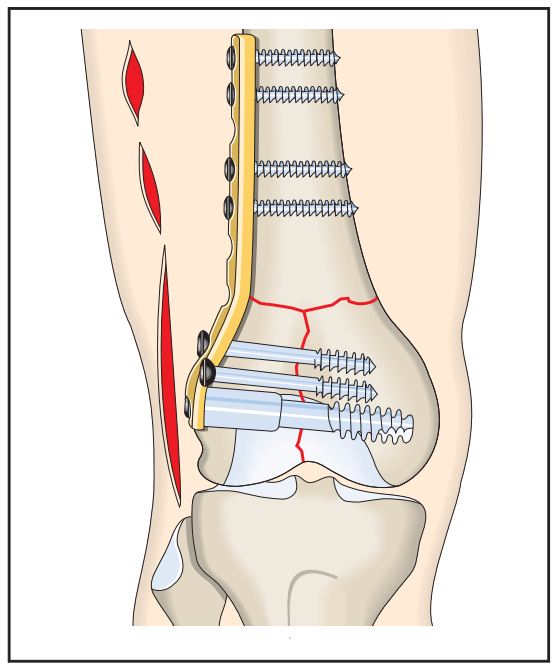

Abb. 5 DCS (1985). Die winkelstabile Fixation ist mit einer großvolumigen Schraube wie bei der DHS verwirklicht. Auch diese Schraube kann in dem allerdings $90^{\circ}$-Schenkel rutschen. In dieser Richtung wirkt keine Kraft, sodass diese im Grunde genommen keine dynamische Schraube ist. Der Vorteil ist, dass sie wie eine Kondylenplatte wirkt, aber in 2 Schritten implantiert werden kann: Zunächst Setzen der DCS-Schraube in der Oberschenkelkondyle und im 2. Schritt Aufsetzen und Platzieren der Platte. Dieses Zusammensetzen hat dazu geführt, dass die DCS am Beginn der minimalinvasiven Osteosynthese Anwendung gefunden hat, weil man nur die kurze Hülse der Platte auf die Schraube heben musste.
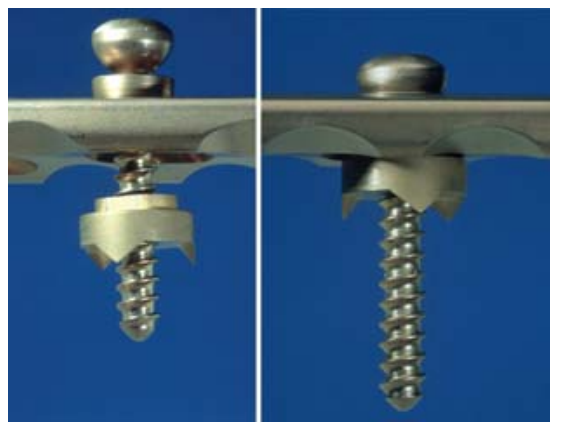

Abb. 6 Shuli (1985). Bei den Shuli handelt es sich um eine Unterlegscheibe mit Gewinde, welche genau in ein DCP-Loch von unten, $d$. h. von Kortikalisseite, eingepasst werden kann. Dadurch erreicht man mit jeder konventionellen DCP-Platte und konventionellen Schraube eine winkelstabile Schraube. Leider hat diese „winkelstabile Konterscheiben-SchraubenTechnik“ ein Schattendasein geführt. In geeigneter Konstellation kann diese aber heute noch gute Dienste leisten. Der Autor unterlässt eine Diskussion, warum diese winkelstabile Unterlegscheibe womöglich zu kompliziert in der Anwendung war - obwohl sie es gar nicht ist!
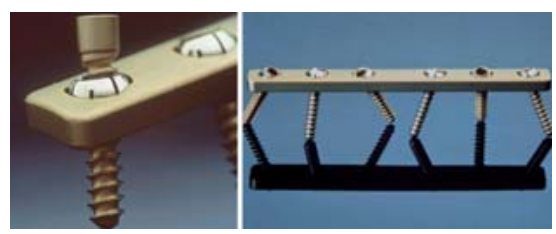

Abb. 8 Kugelkopfschraube/Platte (1988). In den späten 80er-Jahren hat sich der Gedanke bei Entwicklern und Ärzten durchgesetzt, dass winkelstabile Schrauben ein großer Vorteil sein könnten. Auch die Polyaxialität war als Vorteil erkannt worden. Dies fand Ausdruck in einem Prototyp: eine Platte hatte in ihren Löchern eine spreizbare Kugel, in welche eine Schraube mit konischem Kopf eingetaucht ist. Das Eintauchen der Schraube hat die Kugel dann verpresst. Dadurch ist bzw. wäre eine winkelstabile polyaxiale Schrauben-PlattenKonstruktion entstanden. Diese Konstruktion ist nicht marktreif geworden, weil sie bereits aus damaliger Sicht für den Markt zu teuer geworden wäre.

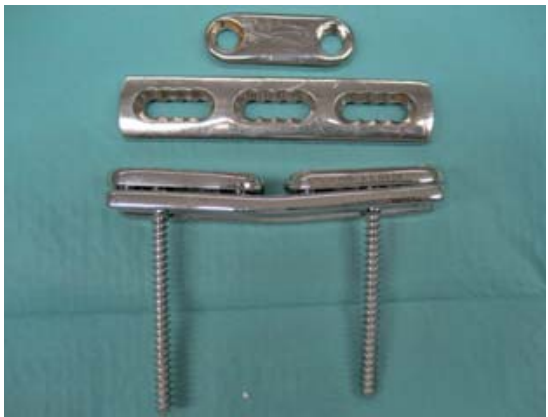

Abb. 7 Druckplattenfixateur (1987). Von Wolters und Gerngross wurde eine Platte entwickelt, die folgendermaßen funktioniert: Eine weitgehend konventionelle Platte wurde mit konventionellen Schrauben bestückt. Die Schraubenköpfe ragen etwas über die Oberfläche hinaus. Gegen diesen herausstehenden Teil der Schraube wird eine Platte gepresst, welche Einmuldungen hat, die genau negativ den Plattenkopf abbilden. Diese Platte wird dann gegen die Grundplatte verschraubt. Ein kleiner Spalt erlaubt es, dass dieses Heranpressen den Schraubenkopf in sein Bett auf der Grundplatte presst und von oben ebenfalls gegengehalten wird, sodass eine in einem bestimmten Winkel mögliche Polyaxialität winkelstabil blockiert wird. Anwendung hat dies z.B. als winkelstabiler Fixateur interne an der Wirbelsäule gefunden (Druckplattenfixateur). Aber im Einzelfall war auch eine Positionierung am Schaftknochen möglich. Dieses Prinzip hat dann später bei der winkelstabilen Platte mit aufschraubbarem Deckel Anwendung und weite Verbreitung gefunden (siehe Abb. 14) (NCB-System). 


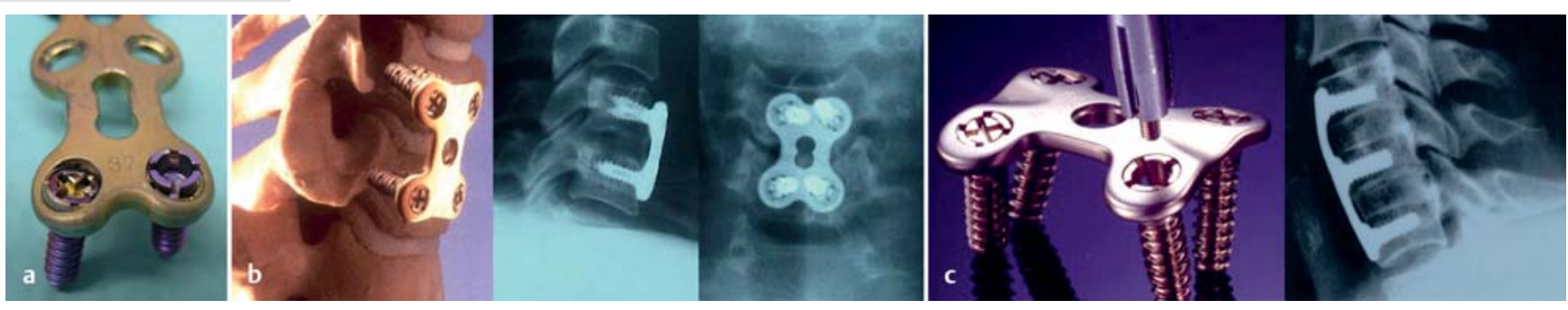

Abb. 9 Winkelstabile Halswirbelsäulenplatte (1989). Die auch heute noch vielfach genutzte ventrale HWS-Platte funktioniert ein wenig nach demselben Prinzip wie bei Abb. 8. In die HWS-Platte wird eine Schraube eingebracht, deren Kopf in 4 Segmente geteilt ist. Die Schraube kann in einem geringen Winkel polyaxial eingebracht werden. Bei der endgültigen Position wird dann in das Zentrum des viergeteilten Kopfes eine Madenschraube eingedreht, welche wiederum diesen viergeteilten Kopf nach außen gegen die Wand der Platte presst.

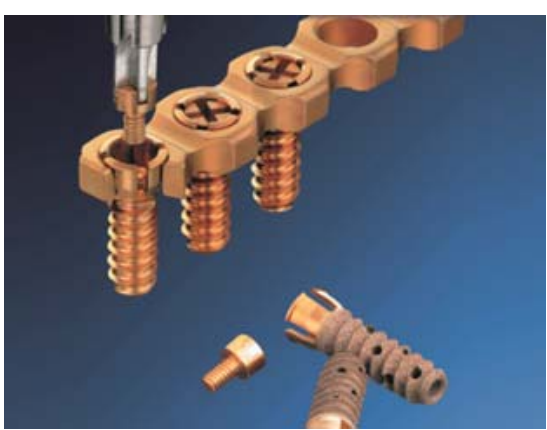

Abb. 10 THORP-Prinzip (1990). Die Wirbelsäulenoperateure (s.o. HWS-Platte) und die gesichtschirurgischen Operateure waren in dieser Zeit wohl etwas konsequenter. Für den mund-kiefer-gesichtschirurgischen Bereich gab und gibt es eine lange winkelstabile Platte, die nach dem ähnlichen Prinzip arbeitet. Ein viergeteilter Schraubenkopf wird nach endgültiger Positionierung durch eine zentrale Madenschraube nach außen gegen die Wand der Platte expandiert, sodass eine stabile Blockade entsteht.
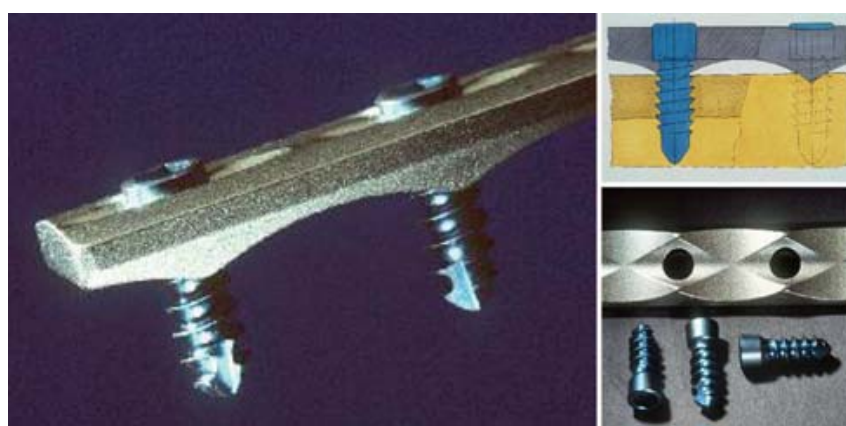

Abb. 11 PCFix (1992). Beim PCFix wurde die Winkelstabilität durch einen konischen Kopf in einem identisch konischen Loch in der Platte erreicht. Die Stabilisierung ist mit einem Konusverschluss aus Chemieund Apothekenflaschen bekannt. Auch die Werkzeuge in Werkzeugmaschinen wurden und werden mit solchen Koni gehalten (dort ein uraltes Prinzip). Die PCFix-Platte hatte dann noch eine andere Besonderheit. Sie hat den Kontakt zum so wertvollen Periost des Knochens auf nur noch Punktkontakte reduziert. Diese Platte wurde für das 3,5mm-System ausgelegt. Fehlender Enthusiasmus am Markt und dann die Entwicklung des etwas toleranteren gewindetragenden Verriegelungsschraubensystems haben dann die Anwendung auslaufen lassen.

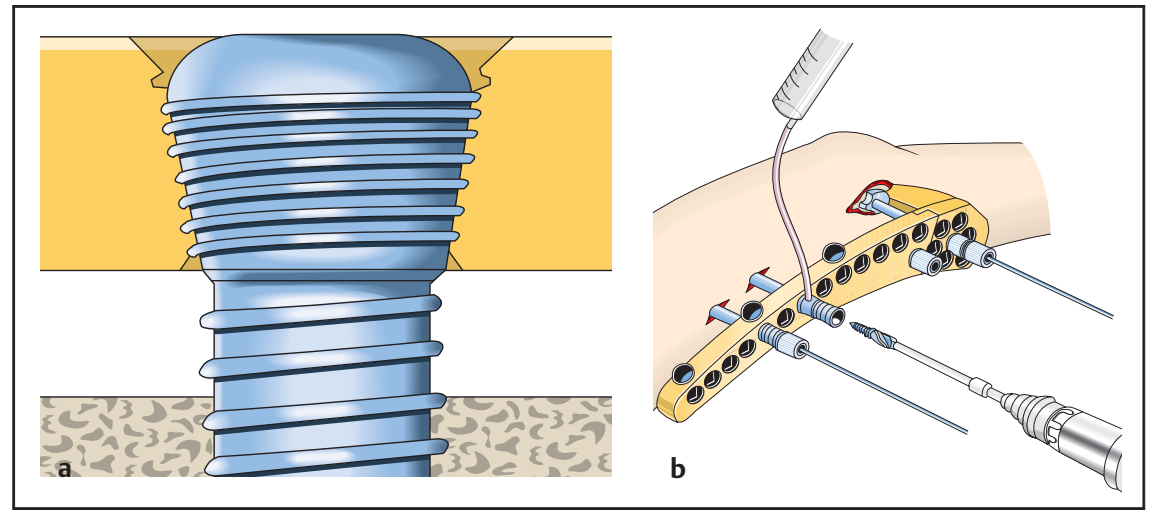

Abb. 12 LISS/LCP (1992). Prinzip des gewindetragenden, winkelstabilen Schraubenlochs ist: Ein konisches Gewinde in der Platte wird mit einem ebenso konischen Schraubenkopf besetzt. Im Grunde genommen könnte man hier eine unendlich feste Stabilisierung erreichen, allerdings dann auch ein Verklemmen, Blockieren oder Kaltverschweißen. Deshalb müssen diese Schrauben mit einem Drehmomentbegrenzer eingebracht werden. Eine Anmerkung nebenbei: Im Grunde genommen hat jede technische Schraube ein bestmögliches Andrehmoment. Zu viel und zu wenig sind von Schaden. Anwendung hat dieses LCP-Prinzip zunächst in der LISS-Platte gefunden. Es wird Folgendes erreicht: Die Stabilisierung ist winkelstabil. Für Kondylen, Tibiakopf und proximalen Femur entspricht die Haltewirkung den Kondylenplatten. Sie ist anatomisch vorgeformt. Sie muss und darf nicht auf den Knochen aufgepresst werden. Dadurch wird das Periost geschont und sie kann minimalinvasiv eingeschoben werden, weil der exakte Knochenkontakt auf der gesamten Strecke nicht angepasst werden muss. Minimalinvasive Osteosynthesen (ein anderes Thema) haben ihren Siegeszug mit diesen winkelstabilen Platten angetreten. Beispiel der Anwendung finden Sie in den anderen Artikeln dieses Heftes. 


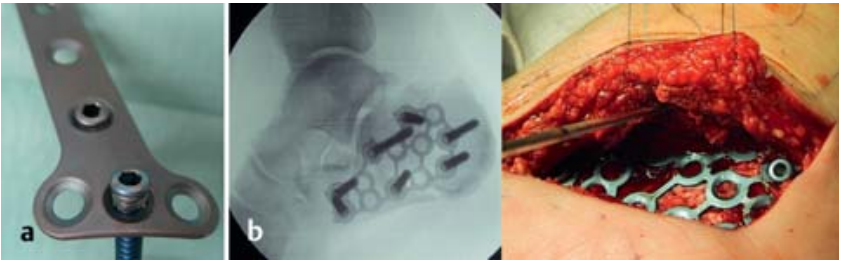

Abb. 13 LITOS-System (1994), von Wolters entwickelt und eingeführt. Eine gewindetragende Schraube trifft auf ein Plattenloch in einer Platte mit geringfügig weicherer Legierung als die Schraube. Das Schraubenloch hat noch kein Gewinde. Beim Vordrehen der Schraube mit etwas härterem Titan drängt sich (nicht schneiden) das Gewinde von selbst ein. Eine Lippe am Anfang des Loches erleichtert dieses Einlaufen. Das Schraubenloch erlaubt einen polyaxialen Winkel. Sehr dünne Platten waren damit möglich. Dies war und ist z. B. ein Vorteil am distalen Radius oder am Kalkaneus. Im Laufe der Jahre hat dieses System dann weitere Verbesserungen erfahren.

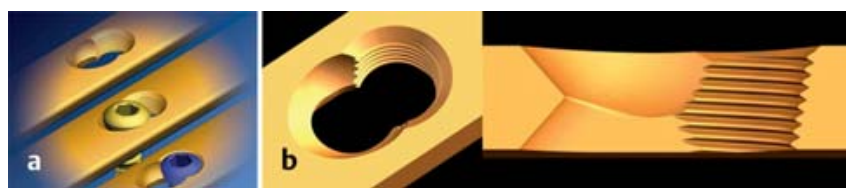

Abb. 15 Combihole-Prinzip (2002) von Frigg und Wagner. Es wurde ein Kombinationsloch entwickelt: 2 Löcher liegen so nah beieinander, dass sie sich teilweise überdecken und in der Silhouette eine 8 (Acht) bilden. Jedes Loch hat ungefähr $2 / 3$ seines Umfangs. Das mittlere Drittel überdeckt sich jeweils. Ein Loch ist nun wie ein DCP-Prinzip aufgebaut, das andere wie ein LCP-Prinzip. Zwei Drittel des Umfangs des jeweiligen Plattenlochdesigns reichen aus, um die Eigenschaften voll zu erhalten. So ist es möglich, in dieses Kombinationsloch (Combihole) entweder eine konventionelle Schraube (nach dem dynamischen DC-Prinzip oder neutral) oder eine winkelstabile Schraube einzubringen.

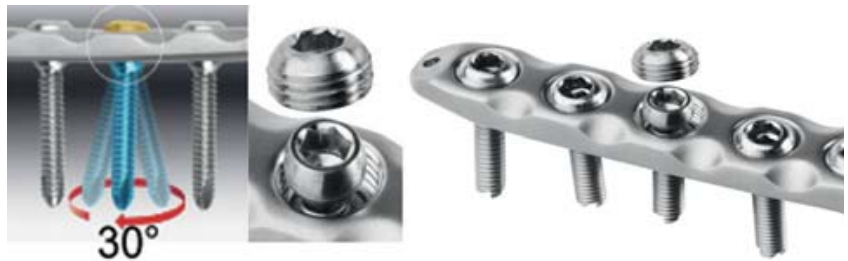

Abb. 14 NCP-System (1996) von Zimmer. Das Druckplattensystem von Wolters hat später Serienreife für lange Platten erreicht. Jedes Plattenloch hat ein Bett um den Schraubenkopf aufzunehmen. Gleichzeitig hat das Plattenloch ein zusätzliches Innengewinde. Ein Deckel mit entsprechendem Außengewinde kann so aufgeschraubt werden, dass der Schraubenkopf gegen den Untergrund und der Deckel gegen die Außenwölbung der Schraube gepresst wird. Beides führt dazu, dass die Schraube in der Platte winkelstabil blockiert ist. Verschiedene Winkel sind möglich, d.h. Polyaxialität ist gegeben. Der Vorteil ist, die Platte kann offen oder eingeschoben eingebracht werden. Es können konventionelle Schrauben verwendet werden. Diese können dann je nach Wunsch des Operateurs blockiert werden.

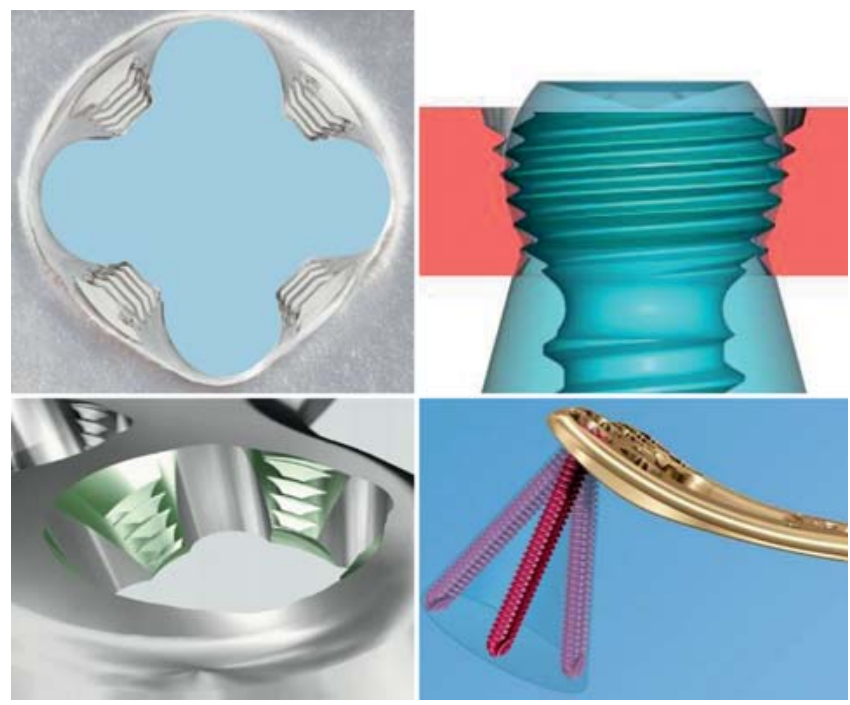

Abb. 16 Polyaxiale Lösungen für Gewindekopfschrauben (2008). Neben den schon genannten Lösungen für Polyaxialität kann auch die über einen Gewindekopf verriegelte Schraube polyaxial ausgelegt werden: Hierzu wird das Gewinde kleeblattförmig unterbrochen. Der Gewindekopf der Schraube wird etwas modifiziert. Der Konus zeigt eine leichte Rundung statt eines geraden Konus. Auf diese Weise ist es möglich, den gewindetragenden Schraubenkopf genau $90^{\circ}$ in das Gewinde einzubringen oder man kann den Schraubenkopf kippen. Dann läuft das Gewinde des Schraubenkopfs in dem Kleeblattgewinde um 1, 2 oder 3 "Stockwerke“ tiefer oder höher versetzt. So können Winkel in einem Kreis von $\pm 15^{\circ}$, also insgesamt $30^{\circ}$, erreicht werden. 


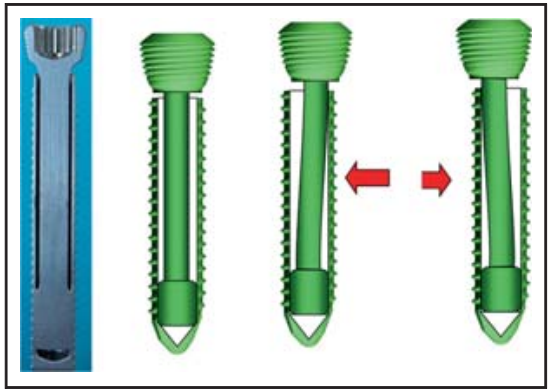

Abb.17 Dynamische Kopfverriegelungsschrauben - DLS (2011/12). Winkelstabile Platten-/ Schraubensysteme sind besonders „unter“ der Platte rigide. Dies trifft auch für konventionelle Platten zu, wenn die Schrauben ihre Aufgabe erfüllen und es zu einem stabilen Anpressen der Platte an den Knochen kommt. Die Frakturheilung wünscht im Grunde genommen eine physiologische elastische Stabilität. Dies kann auch dadurch erreicht werden, dass eine etwas längere Schwingstrecke eingehalten wird und die frakturnahen Schrauben nicht besetzt werden. Bei den Platten handelt es sich dann aber immer noch um eine Schwing- bzw. Kippbewegung. Ein neuer Schritt zur auch unter der Platte elastischen Stabilität mit winkelstabilen Schrauben kann auf zweierlei Weise erreicht werden: 1. Die Schraube hält nur in der Gegenkortikalis und in der zugewandten Kortikalis kann sie schwingen (auf englisch: far-cortex-stabilisation). Diese Art von Verschraubung verzichtet aber auf die Haltekraft der zugewandten Kortikalis. Wir wissen, dass Schrauben in nur einer Kortikalis schlecht stabilisiert sind, da allein von den Hebelkräften die Arbeitslänge fehlt. Bei dieser Lösung ist es nur eine Kortikalisstärke. Bei Schrauben in 2 Kortikales ist die Arbeitslänge so lang wie der Knochen dick ist, also ungefähr das Zehnfache. 2. Die technisch konsequentere Lösung ist eine Schraube, in welche die dynamische Federung eingebaut ist. Dies ist bei der dynamischen Verriegelungsschraube (DLS) dadurch erreicht, dass in einem Zylinder, welcher das Außengewinde trägt, der kopfverriegelte Schraubenkopf über einen Stift in der Außengewindehülse verankert ist (siehe Kapitel DLS).

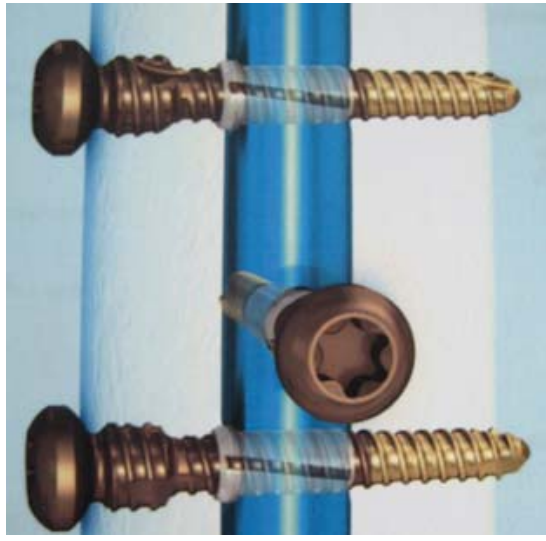

Abb. 20 ASLS winkelstabile Verriegelung von Marknägeln mit Angular Stable Locking System (2009). Es können konventionelle Nägel verwendet werden. Die Schraube trägt eine Kunststoffhülse, welche über einen ansteigenden Durchmesser der Verriegelungsschraube im Nagel zentripedal expandiert und somit wie ein „Fischerdübel“ die Schraube im Nagel winkelstabil belastbar hält. Wie bei einem Dübel muss dazu die zugewandte Kortikalis so weit aufgebohrt werden, dass der Dübel durchpasst. Die ASLS-Schrauben haben dann unter dem Kopf ein größeres Gewinde, welches genau in dieses größere zugewandte Loch hineingeschraubt wird. Somit hält diese Verriegelungsschraube 1 . in beiden Kortikales und 2. winkelstabil im Nagel.
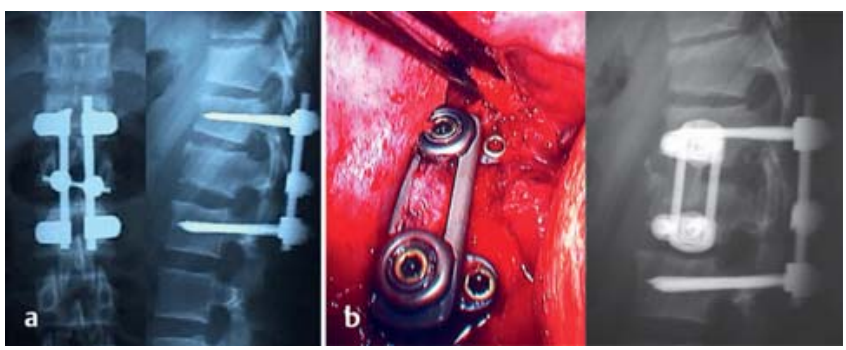

Abb. 18 Winkelstabiler Fixateur interne von dorsal (a), winkelstabiles System von ventral (b).

Interessenkonflikt: Der Unterzeichner ist Mitglied der AO, AOTK Gremien, awiso, Beratung von Synthes, Ceramtex, GisaMed, Vision Medical.

\section{Prof. Dr. med. Dankward Höntzsch}

Leitender Arzt

Abteilung für Medizin-Technische Entwicklungen

BG-Unfallklinik Tübingen

Schnarrenbergstraße 95

72076 Tübingen

hoentzsch@t-online.de

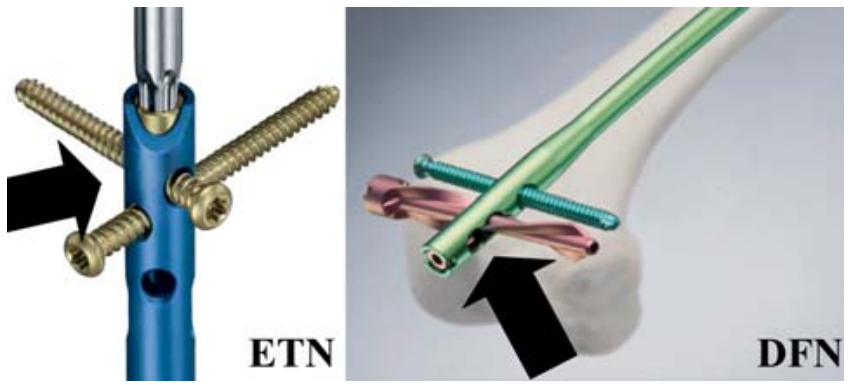

Abb. 19 DFN und ETN. Auch Marknägel können winkelstabil verriegelt sein (1980). Hierbei ist es so, dass die insertionsnächste Verriegelungsschraube oder eine Spiralklinge durch eine zentral eingebrachte Madenschraube blockiert wird (DFN, ETN, proximalste Schraube und UHN). Oder die Nägel (dann aber teuer und speziell angefertigt) tragen ein Gewinde (problematisch, dass der Gewindegang genau getroffen wird und mit dem Gewinde in der Kortikalis korreliert) oder es werden Kunststoffringe eingebaut. Eine konsequente technische Lösung ist in Abb. 20 dargestellt.

\section{Stephan Perren}

$\mathrm{AO}$

Clavadelerstrasse
7500 Davos
Schweiz

Röbi Frigg

CTO Synthes

Luzernstrasse

4500 Solothurn

Schweiz 